\title{
PEDIATRIC
}

\section{Clinicoradiologicial aspects of secretory carcinoma breast: A rare pediatric breast malignancy}

\author{
Aanchal Bhayana, Ritu N Misra, Sunil K Bajaj, Himani Bankhar \\ Department of Radiodiagnosis, Vardhman Mahavir Medical College and Safdarjung Hospital, New Delhi, India \\ Correspondence: Dr. Ritu N Misra, Department of Radiodiagnosis, Vardhman Mahavir Medical College and Safdarjung Hospital, \\ New Delhi - 110 029, India. E-mail: misraritu@gmail.com
}

\begin{abstract}
Secretory carcinoma (juvenile carcinoma) is one of the very rare breast malignancy reported to be prevalent in pediatric age group. We report imaging and clinicopathological features of secretory carcinoma breast with distant and axillary metastasis, in an 11-year-old girl, who presented with a painful lump in right breast. Ultrasound revealed a well-defined, partially microlobulated hypoechoic mass with skin and pectoralis muscle involvement and a suspicious morphology right axillary lymph node. Color Doppler revealed increased vascularity in both the breast mass and suspicious axillary node. Magnetic resonance imaging helped in better evaluation of pectoralis muscle involvement. Computed tomography (CT) neck, chest, and abdomen revealed multiple fibronodular opacities in bilateral lung fields. 18 Flouro-Deoxy-Glucose Positron Emission Tomography (FDG PET-CT) showed a hypermetabolic retroareolar breast mass with multiple hypermetabolic bilateral lung nodules suggesting lung metastasis. The histopathology confirmed the diagnosis of secretory carcinoma. The patient was offered chemotherapy for 2 years and put on follow-up since then.
\end{abstract}

Key words: Axillary metastasis; distant metastasis; imaging features; juvenile carcinoma; pediatric breast malignancy; secretory carcinoma breast

\section{Introduction}

The spectrum of conditions that affects pediatric breast differs significantly from that of adults. The majority of breast masses in children and adolescents are benign, fibroadenoma being the commonest diagnosis. ${ }^{[1]}$ The malignant breast masses in children are exceedingly rare, most of them being the metastatic or disseminated lesions. ${ }^{[1-3]}$ Secretory carcinoma is a rare primary breast malignancy in pediatric age group. ${ }^{[1]}$ Various investigators in past have reported the clinicopathological characteristics, but there is paucity of literature on

\begin{tabular}{|l|l|}
\hline \multicolumn{2}{|c|}{ Access this article online } \\
\hline Quick Response Code: & \\
\hline & Website: \\
\hline
\end{tabular}

its imaging features. ${ }^{[4-7]}$ We report a case of secretory carcinoma breast, in an 11-year-old girl, with distant and axillary metastasis. Distant metastases from secretory carcinoma are extremely rare with only five cases reported, with ours being the sixth case, to the best of our knowledge ${ }^{[8]}$ Moreover, this report is among the very few published reports, where the imaging features of pediatric secretory carcinoma breast are emphasized, on various imaging modalities including ultrasound, color Doppler, computed tomography (CT), magnetic resonance imaging (MRI), and PET-CT. ${ }^{[6-8]}$

This is an open access journal, and articles are distributed under the terms of the Creative Commons Attribution-NonCommercial-ShareAlike 4.0 License, which allows others to remix, tweak, and build upon the work non-commercially, as long as appropriate credit is given and the new creations are licensed under the identical terms.

For reprints contact: reprints@medknow.com

Cite this article as: Bhayana A, Misra RN, Bajaj SK, Bankhar H. Clinicoradiologicial aspects of secretory carcinoma breast: A rare pediatric breast malignancy. Indian J Radiol Imaging 2018;28:448-51. 


\section{Case Report}

An 11-year-old girl, presented to Surgery OPD, in 2014, with a painful lump and watery discharge from right breast for 3-4 months. No significant past history was obtained. There was a palpable firm to hard lump, in retroareolar area of right breast, adherent to the overlying nipple, and underlying pectoralis muscles, with palpable right axillary lymphadenopathy. Provisional diagnosis of suspicious malignant right breast mass with axillary lymphadenopathy was made. After obtaining informed consent from the parents, Ultrasound bilateral breasts was performed, which showed normal left breast [Figure 1A]. The right breast depicted a well-circumscribed retroareolar, oval shaped, homogeneously hypoechoic mass, with partially microlobulated margins, and no internal calcifications or necrosis or dirty edge shadows [Figure 1B]. The mass was seen to extend into skin anteriorly and abutting pectoralis major muscle posteriorly [Figure 1C]. Ultrasound right axilla showed a round hypoechoic node with no fatty hilum [Figure 1D]. Color Doppler showed increased central and peripheral vascularity in the mass and abnormal axillary node [Figure 2A and B]. Diagnosis was made of a suspiciously malignant right breast mass, with likely invasion of skin and pectoralis muscle with malignant right axillary lymphadenopathy. MRI bilateral breasts was performed for better assessment of pectoralis involvement, which revealed an irregular heterogeneously enhancing retroareolar right breast mass with infiltration of skin and subcutaneous tissue. However, the intervening fat plane with the pectoralis major was maintained [Figure 3]. The mass lesion showed a T1 hypointense and T2 heterogeneously hyperintense signal intensity on MRI. US guided Biopsy showed tumor cells in diffuse sheets with minimal pleomorphism, laden with

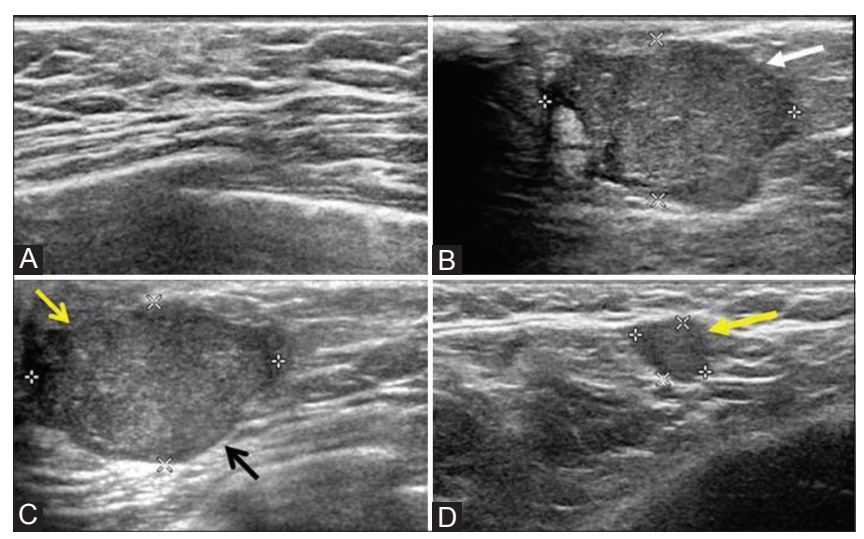

Figure $1(A-D)$ : Ultrasound images of bilateral breasts $(A-C)$, show normal left breast $(A)$, with a well circumscribed, retroareolar, oval-shaped, homogeneously hypoechoic mass, in the right breast (white arrow), with partially microlobulated margins and no internal calcifications or necrosis or dirty edge shadows (B). The right breast mass is seen to extend into the skin anteriorly (yellow open arrow) and abutting pectoralis major muscle posteriorly (black arrow) (C). Ultrasound image of right axilla (D) shows a round hypoechoic node with no fatty hilum (thick closed yellow arrow)
Periodic Acidic Schiff (PAS)-positive secretions, suggesting secretory carcinoma [Figure 4].

On immunohistochemistry (IHC), the tumor was ER, PR, and HER2-neu negative (Triple Negative). Genetic analysis was negative for $B R C A 1, B R C A 2$ genes. Staging $C T$ revealed multiple bilateral fibro-nodular lung opacities suggesting pulmonary metastasis [Figure 5A-E]. Whole body PET-CT showed hypermetabolic retroareolar right breast mass with multiple bilateral FDG avid lung nodules. Final diagnosis was a triple negative secretory carcinoma, right breast, with axillary and pulmonary metastasis (stage IV disease). The patient was started on palliative chemotherapy. Follow-up CT after 2 years showed residual retroareolar irregular heterogeneously enhancing right breast mass free from overlying skin. CT chest showed post chemotherapy skin edema and reduction in number of lung nodules [Figure 6A-C]. Follow-up PET-CT after 2 years showed a mass free from overlying skin, with multiple bilateral lung nodules, showing mild FDG uptake [Figure 6D]. There was minimal reduction in size of the mass with significant resolution of lung nodules. Following these investigations, the patient is on follow-up.

\section{Discussion}

Carcinoma breast is extremely rare in children $(<1 \%$ of all breast lesions). ${ }^{[3]}$ BRCA1 and BRCA2 gene mutations are associated with pediatric breast cancers. Secretory carcinoma is most common type of cancer seen in children, accounting for $<0.1 \%$ of all invasive breast cancers. ${ }^{[1]}$ Other histological types less frequently encountered in children are medullary, anaplastic, inflammatory, infiltrating lobular, and ductal carcinomas, which are more aggressive, unlike favorable prognosis of secretory carcinoma. ${ }^{[1]}$

Secretory carcinoma was initially termed as juvenile breast carcinoma by McDivitt and Stewart in 1966, owing to presentation, in young children. ${ }^{[9]}$ Subsequently, Tavassoli and Norris coined the term "secretory carcinoma" based on typical histopathology (vacuolated cytoplasm, intracellular, and extracellular secretory material). ${ }^{[4]}$ On IHC, these carcinomas are usually triple negative (ER, PR, and HER-2/neu negative), with a recently identified balanced translocation $\mathrm{t}(12 ; 15)$ ETV6-NTRK3 fusion gene. ${ }^{[8]}$
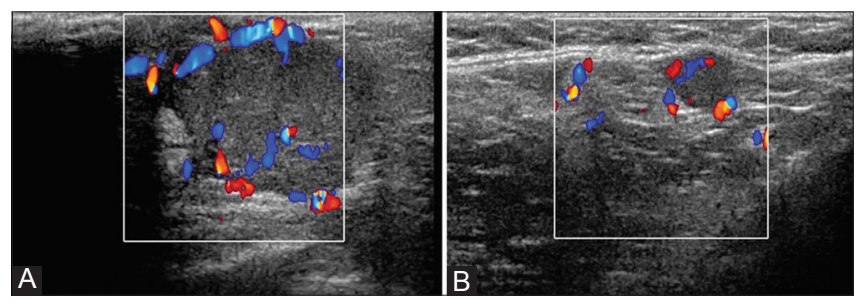

Figure 2 ( $A$ and $B$ ): Color Doppler images ( $A$ and $B$ ) show increased central and peripheral vascularity in the mass $(A)$ and abnormal axillary node $(B)$ 


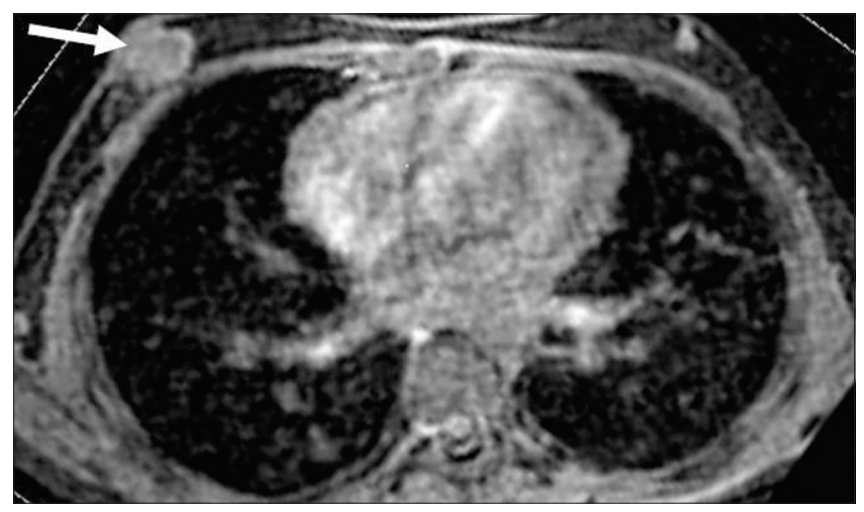

Figure 3: Axial contrast enhanced T1 weighted magnetic resonance imaging image shows an irregular heterogeneously enhancing retroareolar right breast mass (white arrow), with infiltration of skin and subcutaneous tissue anteriorly and maintained fat plane with pectoralis major posteriorly

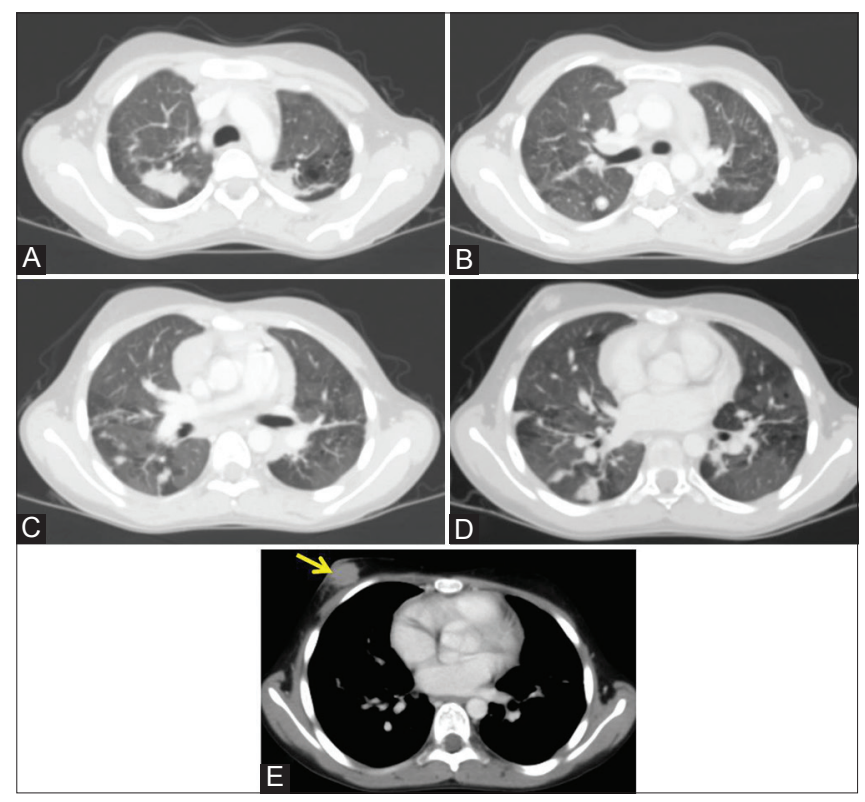

Figure 5 (A-E):Axial contrast enhanced computed tomography chest images in lung window $(A-D)$ and mediastinal window $(E)$ show multiple bilateral fibro-nodular lung opacities $(A-D)$, with a heterogeneously enhancing irregular retroareolar right breast mass (yellow arrow) $(E)$

The patients present with a slow growing, well-circumscribed, retro-areolar mass, with or without nipple discharge. There is lacuna in literature on specific imaging characteristics of secretory breast carcinoma. ${ }^{[6,7]}$

On ultrasound, a solitary, well-circumscribed, or partially microlobulated hypoechoic or isoechoic mass is noted, mimicking a benign lesion. ${ }^{[1,6,7]}$ The role of $\mathrm{CT}$ in pediatric breast masses is limited to staging. ${ }^{[8]}$ MRI is important in assessment of chest wall infiltration.

The differentials in a sonographically solid pediatric breast lesion are benign and malignant etiologies. The benign lesions include fibroadenomas, granular cell tumours, juvenile papillomatosis, and pseudoangiomatous stromal

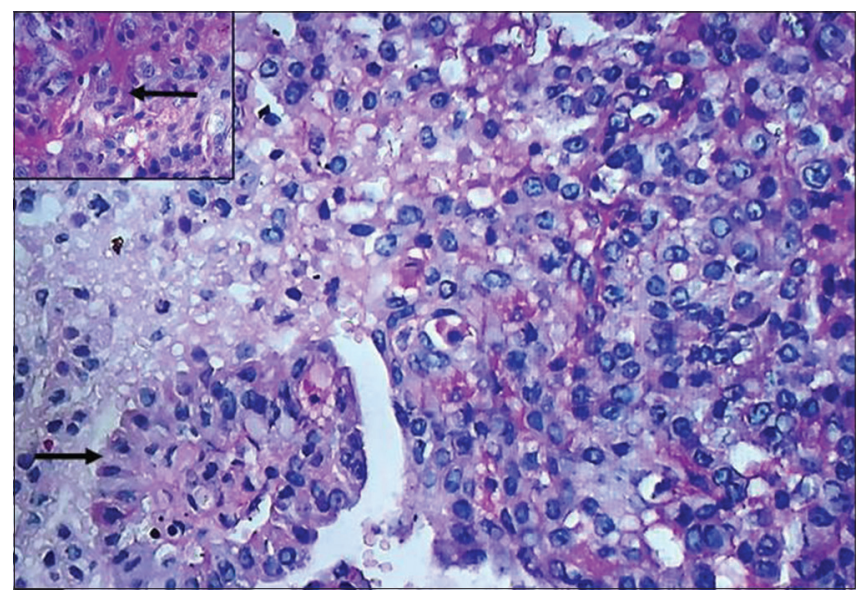

Figure 4: Histopathology section shows tumor cells in diffuse sheets and tubules with minimal pleomorphism (PAS $\times 200$; black arrow). Inset shows PAS-positive secretions $(\times 400$; black arrow)

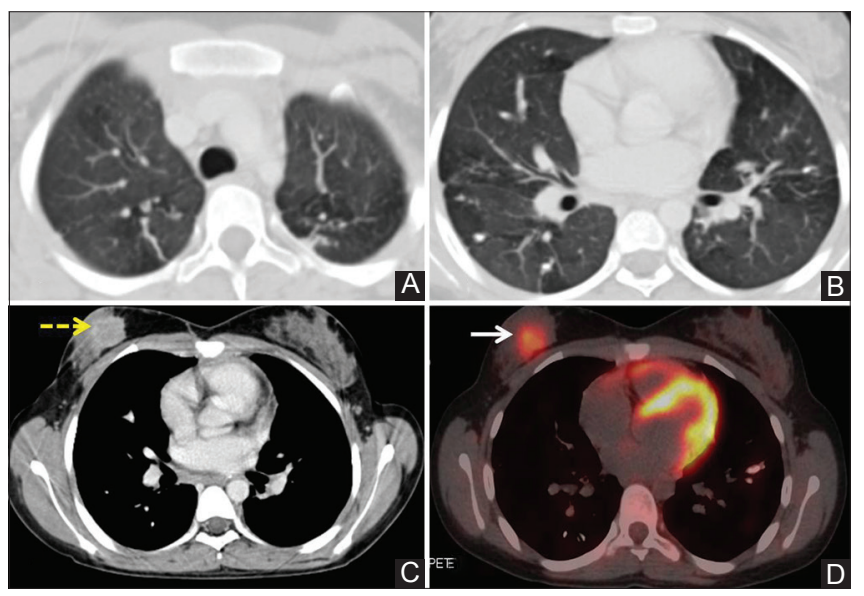

Figure 6 (A-D): Axial contrast enhanced computed tomography chest images (postchemotherapy), in both lung window $(A, B)$ and mediastinal window $(C)$, show reduction in number of bilateral lung nodules $(A-C)$, with an irregular heterogeneously enhancing retroareolar right breast mass (yellow dotted arrow) with minimal reduction in size and postchemotherapy skin edema (C). A 2-year follow-up PET CT image (D) shows mild FDG uptake in both the retroareolar right breast mass and bilateral lung nodules (white arrow). The right breast mass is now seen to be free from overlying skin (D)

hyperplasia (PASH). The malignant lesions include primary breast malignancies like phylloides tumor and carcinomas and breast secondaries. The imaging features of fibroadenomas, $\mathrm{PASH}$, juvenile papillomatosis, and phylloides tumor mimic each other on ultrasound and are seen as well circumscribed homogeneously hypoechoic wider than taller masses. Presence of anechoic cysts and clefts in phylloides may be a distinguishing feature. Juvenile papillomatosis shows multiple tiny peripheral cysts. Granular cell tumor, though a benign tumor, masquerades malignancy on ultrasound, with irregular spiculated hypoechoic mass, and dirty posterior acoustic shadows. Breast carcinomas depict malignant features on sonography. Secretory carcinoma may mimic a benign lesion on ultrasound. However, finally confirmation needs 
histopathology. Sanders et al. have recently laid down guidelines, on when to perform biopsy, if encountered with a pediatric solid breast mass. ${ }^{[1,10,11]}$

There is no consensus on ideal treatment strategy for secretory breast carcinoma. ${ }^{[1,4-8]}$ However, breast conservative surgery (BCS) is considered the mainstay of treatment. Axillary metastasis is rare, with an incidence of about $30 \%$ in children. Involvement of more than three lymph nodes may herald a risk for distant metastasis. ${ }^{[4-7]}$ The role of adjuvant chemotherapy and radiotherapy is not established, though their administration following BCS, improves locoregional control. ${ }^{[5-7]}$ Since, usually secretory tumors are triple negative, effectiveness of hormone therapy is of little use. Recently, the identification of specific ETV6-NTRK3 fusion gene mutation is considered to be a breakthrough in formulating targeted molecular therapies for this disease in the near future. ${ }^{[8]}$

\section{Conclusion}

Since majority of breast lumps, in children mimic fibroadenomas, a high suspicion is required to reach a diagnosis, thereby guiding appropriate management. Secretory carcinoma, though a very rare pediatric breast malignancy, should be considered as a differential diagnosis in slowly growing palpable mass, with solid benign looking lesion on ultrasound. The awareness of possibility of malignancy in these masses can be a great help in reaching an accurate diagnosis.

\section{Declaration of patient consent}

The authors certify that they have obtained all appropriate patient consent forms. In the form the patient(s) has/have given his/her/their consent for his/her/their images and other clinical information to be reported in the journal. The patients understand that their names and initials will not be published and due efforts will be made to conceal their identity, but anonymity cannot be guaranteed.
Financial support and sponsorship

Nil.

\section{Conflicts of interest}

There are no conflicts of interest.

\section{References}

1. Chung EM, Cube R, Hall GJ, González C, Stocker JT, Glassman LM. Breast masses in children and adolescents: Radiologic-pathologic correlation. Radiographics 2009;29:907-31.

2. Greydanus DE, Matytsina L, Gains M. Breast disorders in children and adolescents. Prim Care 2006;33:455-502.

3. Pettinato G, Manivel JC, Kelly DR, Wold LE, Dehner LP. Lesions of the breast in children exclusive of typical fibroadenoma and gynecomastia: A clinicopathologic study of 113 cases. Pathol Annu 1989;24:296-328.

4. Tavassoli FA, Norris HJ. Secretory carcinoma of the breast. Cancer 1980;45:2404-13.

5. Sharma V, Anuragi G, Singh S, Patel P, Jindal A, Sharma RG. Secretory carcinoma of the breast: Report of two cases and review of the literature. Case Rep Oncol Med 2015;2015. doi: $10.1155 / 2015 / 581892$.

6. Lee SG, Jung SP, Lee HY, Kim S, Kim HY, Kim I, et al. Secretory breast carcinoma: A report of three cases and a review of the literature. Oncol Lett 2014;8:683-6.

7. Mun SH, Ko EY, Han BK, Shin JH, Kim SJ, Cho EY. Secretory carcinoma of the breast sonographic features. J Ultrasound Med 2008;27:947-54

8. Arce C, Cortes-Padilla D, Huntsman D, Huntsman DG, Miller MA, Dueñnas-Gonzalez A, et al. Secretory carcinoma of the breast containing the ETV6-NTRK3 fusion gene in a male: Case report and review of the literature. World J Surg Oncol 2005;3:35.

9. McDivitt RW, Stewart FW. Breast carcinoma in children. JAMA 1966;195:388-90.

10. Karl SR, Ballantine TV, Zaino R. Juvenile secretory carcinoma of the breast. J Pediatr Surg 1985;20:368-71.

11. Sanders LM, Sharma P, El Madany M, King AB, Goodman KS, Sanders AE. Clinical breast concerns in low-risk pediatric patients: Practice review with proposed recommendations. Pediatr Radiol 2018;48:186-95. 